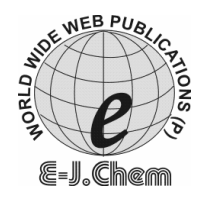

ISSN: 0973-4945; CODEN ECJHAO

E-Journal of Chemistry

http://www.e-journals.net

2009, 6(1), 61-70

\title{
Rapid Biosynthesis of Silver Nanoparticles Using Culture Supernatant of Bacteria with Microwave Irradiation
}

\author{
N. SAIFUDDIN*, C. W. WONG and A. A. NUR YASUMIRA, \\ Chemistry Unit, Department of Engineering Sciences, \\ College of Engineering Universiti Tenaga Nasional, \\ 43900 Serdang, Selangor, Malaysia. \\ saifuddin@uniten.edu.my
}

Received 29 May 2008: Accepted 20 July 2008

\begin{abstract}
The development of rapid and reliable processes for the synthesis of nanosized materials is of great importance in the field of nanotechnology. Synthesis of silver nanoparticles using microorganism have been reported, but the process is rather slow. In this paper, we describe a novel combinatorial synthesis approach which is rapid, simple and "green" for the synthesis of metallic nanostructures of noble metals such as silver (Ag), by using a combination of culture supernatanant of Bacillus subtilis and microwave (MW) irradiation in water in absence of a surfactant or soft template. It was found that exposure of culture supernatanant of Bacillus subtilis and microwave irradiation to silver ion lead to the formation of silver nanoparticles. The silver nanoparticles were in the range of $5-60 \mathrm{~nm}$ in dimension. The nanoparticles were examined using UV-Visible Spectroscopy, and Transmission Electron Microscopy (TEM) analyses. The formation of nanoparticles by this method is extremely rapid, requires no toxic chemicals and the nanoparticles are stable for several months. The main conclusion is that the bio-reduction method to produce nanoparticles is a good alternative to the electrochemical methods.
\end{abstract}

Keywords: Silver nanoparticles, Bacillus subtilis, Culture supertanant, Microwave irradiation, Bio-reduction

\section{Introduction}

Nanotechnology provides the ability to engineer the properties of materials by controlling their size, and this has driven research toward a multitude of potential uses for nanomaterials. Metallic nanoparticles exhibit unusual optical, thermal, chemical, and physical properties. The reduction of materials' dimension has pronounced effects on the 
physical properties that may be significantly different from the corresponding bulk material. Some of the physical properties exhibited by nanomaterials are due to (i) large surface atom, (ii) large surface energy, (iii) spatial confinement, and (iv) reduced imperfections.

One key aspect of nanotechnology concerns the development of rapid and reliable experimental protocols for the synthesis of nanomaterials over a range of chemical compositions, sizes, high monodispersity and large scale production. A variety of techniques have been developed to synthesize metal nanoparticles, including chemical reduction using a number of chemical reductants including $\mathrm{NaBH}_{4}, \mathrm{~N}_{2} \mathrm{H}_{4}, \mathrm{NH}_{2} \mathrm{OH}$, ethanol, ethylene glycol and $\mathrm{N}, \mathrm{N}$-dimethyformamide $(\mathrm{DMF})^{1-5}$, aerosol technique ${ }^{6}$, electrochemical or sonochemical deposition ${ }^{7,8}$, photochemical reduction ${ }^{9}$, and laser irradiation technique ${ }^{10}$. A lot of interest has been created by the term "green nanotechnology". In a broad sense, this term includes a wide range of possible applications, from nanotechnology-enabled, environmentally friendly manufacturing processes that reduce waste products (ultimately leading to atomically precise molecular manufacturing with zero waste); the use of nanomaterials as catalysts for greater efficiency in current manufacturing processes by minimizing or eliminating the use of toxic materials (green chemistry principles); the use of nanomaterials and nanodevices to reduce pollution (e.g. water and air filters); and the use of nanomaterials for more efficient alternative energy production (e.g. solar and fuel cells). With the flourishing demand of "green" nanoparticle synthesis processes" ${ }^{11}$, the field of nanoparticle synthesis has recently developed new routes. These include employing microorganisms, such as: Pseudomonas stutzeri ${ }^{12}$ Verticilium $s p^{13}$, Fusarium oxysporum $^{14,15}$, Thermomonospora $s p^{16}$ and, also, alfalfa and geranium plants ${ }^{17,18}$.

Biosynthetic methods employing either biological microorganisms or plant extracts have emerged as a simple and viable alternative to chemical synthetic procedures and physical methods ${ }^{14-21}$. Although it is known that microorganisms such as bacteria, yeast and now fungi play an important role in remediation of toxic metals through reduction of the metal ions, this was considered interesting as nanofactories very recently. The presence of hydrogenase in the $F$. oxysporium broth was demonstrated. Some bacteria reduce $\mathrm{Fe}^{3+}$ oxides by producing and secreting small, diffusible redox compounds that can serve as electron shuttle between the microbe and the insoluble iron substrate ${ }^{22}$.This extracellular enzyme shows an excellent redox properties and it can act as an electron shuttle in the metal reduction. It was evident that electron shuttles or other reducing agents (e.g. hydroquinones) released by microorganisms are capable of reducing ions to nanoparticles ${ }^{23}$. However, biological nanoparticles synthesis generates nanoparticles at a much slower rate. In the studies synthesis of silver nanoparticles using the cell mass of bacteria ${ }^{24-25}$ and fungi ${ }^{13}$ or their leached cell components ${ }^{14}$, the time required completing the reaction ranged from 24 to $120 \mathrm{~h}$; this lengthy reaction is the one major drawback of the biological synthesis. Biological synthesis would have greater commercial viability if the nanoparticles could be synthesis more rapidly and in the large production scale. Despite stability and a green method of production, particles are not monodispersible and the rate at which they form is not comparable to chemical synthesis methods.

Thermal factors have been demonstrated to affect the size and uniformity of nanoparticles ${ }^{26}$. Controlling particle growth rate is possible through knowledge of a material's properties and controlling of the reaction by changes to the time and temperature. The ability of any material to absorb microwave energy is expressed by its dielectric loss factor combined with the dielectric constant. The microwave radiation heats up a material through its dielectric loss, which converts the radiation energy into thermal energy. The main 
challenges frequently encountered by the researchers of nanoparticle synthesis are (i) controlling the particle size/shape and (ii) achieving the monodispersity. The synthesis of nanoparticles with controlled monodispersity is a recent demand by the material developers for the advancement of nanotechnology. Herein, we describe a novel combinatorial synthesis approach which is rapid, simple and "green" for the synthesis of metallic nanostructures of noble metals such as silver $(\mathrm{Ag})$, by using a combination of culture supernatanant of Bacillus subtilis and microwave (MW) irradiation in water in absence of a surfactant or soft template. We have chosen Bacillus subtilis because these bacteria are ubiquitous bacteria commonly found in various ecological niches including soil, water and air which do not have a history of pathogenicity from contact in the environment.

\section{Chemicals, culture media and test organisms}

The bacteria test strain used was Bacillus subtilis, (ATCC 6633) strain was obtained from WARD'S Natural Science USA and all other chemicals and the culture media unless otherwise stated were obtained from Merck, Germany.

\section{Experimental}

Bacillus subtilis stock cultures were maintained by subculturing at monthly intervals. Growth medium used was Luria Broth (LB) medium (1\% Bacto-tryptone [Difco], 0.5\% yeast extract [Difco], $1 \% \mathrm{NaCl}, \mathrm{pH} 7.5$ ). $250 \mathrm{~mL}$ of Luria broth (LB) was prepared, sterilized, and inoculated with fresh batch of the bacteria, Bacillus subtilis. The culture flasks were incubated for $36 \mathrm{~h}$ at $37^{\circ} \mathrm{C}$ with shaking at $150 \mathrm{rpm}$. After incubation period, the cultures were centrifuged at 5,000 rpm for 10 minutes and the supernatant were collected. These supernatant were used as the starting material for synthesis of nanoparticles.

\section{Extracellular biosynthesis of Ag nanoparticles using culture supernatant of bacillus subtilis}

In a typical synthesis of silver nanoparticle extracellularly, $50 \mathrm{~mL}$ aqueous solution of $1 \mathrm{mM}$ silver nitrate $\left(\mathrm{AgNO}_{3}\right)$ was treated with $50 \mathrm{~mL}$ of Bacillus subtilis supernatant solution in a $250 \mathrm{~mL}$ Erlenmeyer flask ( $\mathrm{pH}$ adjusted to 8.5). The whole mixture was put into a shaker at $40^{\circ} \mathrm{C}(200 \mathrm{rpm})$ for 5 days and maintained in the dark. Control experiments were conducted with uninoculated media, to check for the role of bacteria in the synthesis of nanoparticles. The reduction of $\mathrm{Ag}^{+}$ions was monitored by sampling an aliquot $(2 \mathrm{~mL})$ of the solution at intervals of $24 \mathrm{~h}$. and measuring the UV-Vis spectra of the solution. Absorption measurements were carried out on a Genesys $10 \mathrm{UV}$-VIS spectrophotometer. The spectra were recorded at room temperature using a one-centimetre quartz cuvette.

The transmission electron microscopy (TEM) analysis of extracellular synthesized silver nanoparticles were prepared by drop-coating biosynthesized silver nanoparticles solution on carbon-coated copper TEM grids $(40 \mu \mathrm{m} \times 40 \mu \mathrm{m}$ mesh size $)$. Samples were dried and kept under vacuum in desiccators before loading them onto a specimen holder. TEM measurements were performed on a JEOL model 1200EX electron microscope operated at an accelerating voltage at $120 \mathrm{kV}$.

\section{Combinatorial synthesis of nanoparticles using bacterial supernatant and microwave irradiation}

As mentioned above, $50 \mathrm{~mL}$ aqueous solution of $1 \mathrm{mM}$ silver nitrate $\left(\mathrm{AgNO}_{3}\right)$ was treated with $50 \mathrm{~mL}$ of Bacillus subtilis supernatant solution in a $250 \mathrm{~mL}$ Erlenmeyer flask $(\mathrm{pH}$ adjusted to 9.0). The whole mixture was put in a domestic microwave oven (National Model 
$\mathrm{N} \mathrm{N-GD} \mathrm{576M).} \mathrm{The} \mathrm{sample} \mathrm{was} \mathrm{subjected} \mathrm{to} \mathrm{several} \mathrm{short} \mathrm{burst} \mathrm{of} \mathrm{microwave} \mathrm{irradiation} \mathrm{at}$ frequency of $2.45 \mathrm{GHz}$, at power output of about $100 \mathrm{~W}$ in a cyclic mode (on $15 \mathrm{~s}$, off $15 \mathrm{~s}$ ) to prevent overheating as well as aggregation of metals. The irradiation process was conducted for a minimum of 5 up to maximum of 15 cycles. The reduction of $\mathrm{Ag}^{+}$ions was monitored by sampling an aliquot $(2 \mathrm{~mL})$ of the solution after 5, 7, 9, 12 and 15 cycles and measuring the UV-Vis spectra of the solution. Absorption measurements were carried out on a Genesys 10 UV-VIS spectrophotometer. The spectra were recorded at room temperature using a one-centimetre quartz cuvette. Samples for transmission electron microscopy (TEM) were prepared as described previously.

\section{Nitrate reductase assay}

Nitrate reductase converts nitrate to nitrite. The enzyme activity was measured using the method described by Harley ${ }^{31}$ where the activity was measured by putting in the substrate for the enzyme (nitrate) and then measuring the amount of nitrite after $1 \mathrm{~h}$. The net increase in nitrite at $1 \mathrm{~h}$ is the amount of nitate reductase activity. The reagents were: assay medium: $30 \mathrm{mM} \mathrm{KNO}_{3}$ and $5 \%$ propanol in $0.1 \mathrm{M}$ phosphate buffer $\mathrm{pH} 7.5$; nitrite solution: $25 \mu \mathrm{M}$ $\mathrm{NaNO}_{2}$ (Nitrite) solution; nitrite assay reagents: sulfanilamide solution: $1 \%$ (w/v) in $25 \%$ (v/v) $\mathrm{HCl}$ and $\mathrm{N}$-(1-napthy) ethelenediamine dihydrochloride solution (NEED): $0.02 \%(\mathrm{w} / \mathrm{v}$ ) in distilled water.

Five tubes each containing $10 \mathrm{~mL}$ of the Bacillus subtilis culture supernatant, taken from the $120 \mathrm{~h}$ reaction were prepared. Tubes ' $\mathrm{a}$ ' and 'b' (duplicates) were place in a boiling water bath sample to kill the enzyme as soon as $10 \mathrm{~mL}$ assay medium was added (i.e. at time-zero). After boiling 5 minutes, the tubes were kept at room temperature until the nitrite was measured. $10 \mathrm{~mL}$ of assay medium were added to tubes 'c, d, e' (triplicates) and incubate in the dark for 60 minutes before placing the tubes in the boiling water bath for 5 minutes. After the samples had cooled, $5 \mathrm{~mL}$ of sulfanilamide solution was added to each. Then $5 \mathrm{~mL}$ of NEED solution was added and quickly mixed by inverting the tubes. After 20 minutes the absorbance at $540 \mathrm{~nm}$ was measured using a Genesys 10 UV-VIS spectrophotometer. The amount of nitrite found in tubes ' $a$ ' and ' $b$ ' were subtracted from that in tubes 'c, d', and 'e' to calculate the amount produced by enzyme activity during $60 \mathrm{~min}$. Nitrite standard curve was prepared by placing known amounts of nitrite ( 0 to $250 \mathrm{nmol} \mathrm{NO}$ ) followed by $5 \mathrm{~mL}$ of sulphanilamide and $5 \mathrm{~mL}$ of NEED solution. After mixing and incubation for $20 \mathrm{~min}$ at room temperature absorbance was measured at $540 \mathrm{~nm}$. The enzyme activity was calculated based on the increase in nitrite over $60 \mathrm{~min}$ for the amount of sample $(10 \mathrm{~mL})$ and expressed as nmol nitrite/hour $/ \mathrm{mL}$.

\section{Determination of the tryptophan/tyrosine residues}

Presence of tryptophan/tyrosine residues in proteins released in the bacterial culture supernatant was analyzed spectrophotometrically by measurement of absorbance in the $250-300 \mathrm{~nm}$ wavelength regions. The measurements were carried out on a Genesys 10 UV-VIS spectrophotometer. The spectra were recorded at room temperature using a one-centimetre quartz cuvette.

\section{Results and Discussion}

Extracellular biosynthesis of silver nanoparticles using culture supernatant of bacillus subtilis

Extracellular production of silver nanoparticles by the culture supernatants of Bacillus subtilis with aqueous silver nitrate solution, $1 \mathrm{mM}$ was investigated. The picture of test tubes 
of silver nitrate solution after exposure to culture supernatant of Bacillus subtilis is shown in the inset of Figure 1. The appearance brown colour clearly indicates the formation of silver nanoparticles in the reaction mixture ${ }^{19}$. The characteristics brown colour of colloidal silver solution is due to the excitation of surface plasmon vibrations in the nanoparticle and provides a convenient spectroscopic signature of their formation.

Several hydroquinones with excellent redox properties were reported that could be act as electron shuttle in metal reductions ${ }^{22,}{ }^{23}$. Thus, it was evident that electron shuttle or others reducing agents released by Bacillus subtilis are capable of reducing silver ions to silver nanoparticles. On the other hands, the reduction of silver ions did not occur in the absence of bacterial cells. This clearly indicates that the reducing agents that are released into the cultures of the aforementioned Bacillus subtilis are involved in the reduction process.

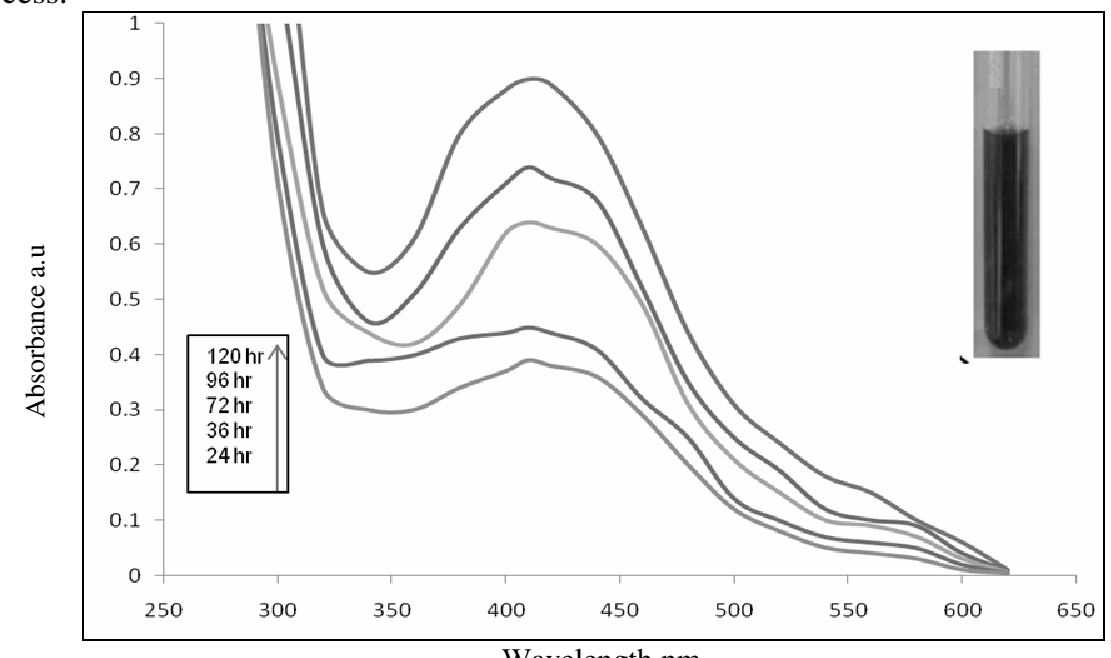

Wavelength $\mathrm{nm}$

Figure 1. UV-Vis spectra recorded as a function of time of reaction of $1 \mathrm{mM}$ aqueous solution of $\mathrm{AgNO}_{3}$ with Bacillus subtilis culture supernatant. The inset of the figure shows a test tube of the silver nanoparticle solution formed at the end of the reaction.

From the UV-Vis spectra recorded for the aqueous silver nitrate - B. subtilis reaction medium as a function of time (Figure 1) it can be observed that the silver surface plasmon band occurs at around $410 \mathrm{~nm}$ and steadily increase in intensity as a function of time of reaction. The surface plasmon band in the silver nanoparticles solution remains close to $410 \mathrm{~nm}$ throughout the reaction period, suggesting that the particles are dispersed in the aqueous solution with no evidence for aggregation. After completion of the reaction, the silver nanoparticles solution was tested for stability. It was observed that the nanoparticle solution of silver was extremely stable for more than six months with no signs of aggregation even at the end of this period. The particles are thus stabilized in solution by the capping agent that is likely to be proteins secreted by the biomass.

A representative TEM picture recorded from the silver nanoparticle film deposited on a carbon coated copper TEM grid is shown in Figure 2. This picture shows individual silver particles as well as a number of aggregates. The morphology of the nanoparticles is highly variable, with spherical and occasionally triangular nanoparticles observed in the micrograph. Under observation of such images in an optical microscope, these assemblies 
were found to be aggregates of silver nanoparticles in the size range 5-50 $\mathrm{nm}$. The nanoparticles were not in direct contact even within the aggregates, indicating stabilization of the nanoparticles by a capping agent. This corroborates with the previous observation by Ahmad et $a l^{14}$ in their study on $F$. oxysporum. As discussed earlier, the silver nanoparticle solution, synthesized by the reaction of $\mathrm{Ag}^{+}$ions with Bacillus subtilis culture supernatant, is exceptionally stable and the stability is likely to be due to capping with proteins secreted by the bacteria. The separation between the silver nanoparticlesseen in the TEM image could be due to capping by proteins and would explain the UV-Vis spectroscopy measurements, which is characteristic of well-dispersed silver nanoparticles.

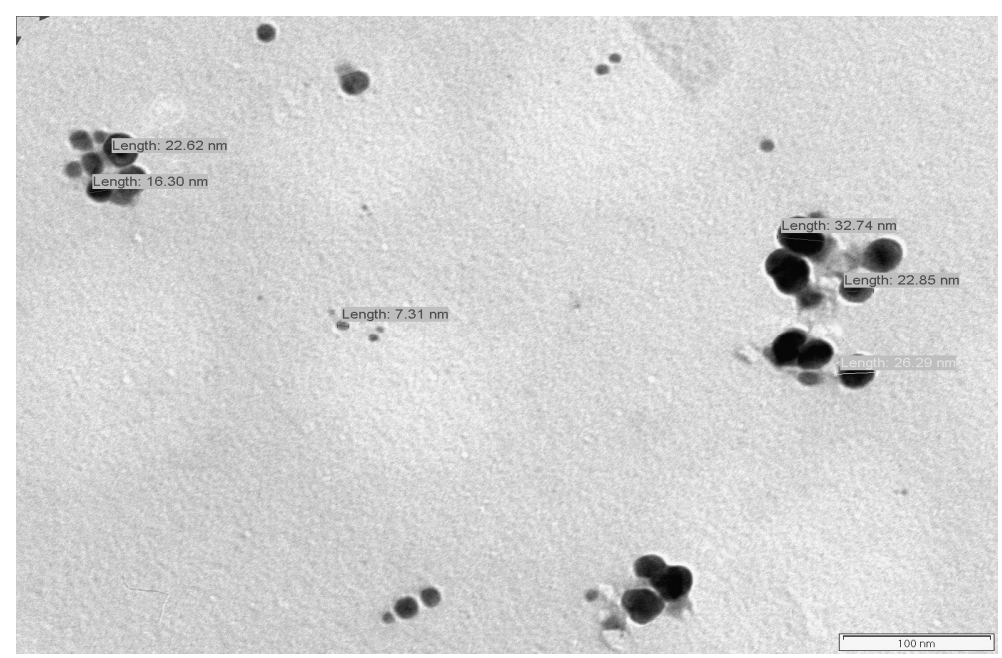

Figure 2. TEM micrograph at 100,000 times magnification recorded from a drop-coated film of an aqueous solution incubated with Bacillus subtilis culture supernatant and reacted with $1 \mathrm{mM} \mathrm{Ag}{ }^{+}$ions for $120 \mathrm{~h}$ at $40^{\circ} \mathrm{C}$. The scale bar corresponds to $100 \mathrm{~nm}$.

\section{Rapid extracellular biosynthesis of silver nanoparticles using culture supernatant of bacillus subtilis and microwave irradiation}

The previous method was extended Rapid extracellular production of silver nanoparticles by the culture supernatants of Bacillus subtilis with aqueous silver nitrate solution was extended by incorporating microwave irradiation. The advantage of using microwave radiation is that it provides uniform heating around the nanoparticles and can assist the digestive ripening of such particles without aggregation. The microwave radiation heats up a material through its dielectric loss, which converts the radiation energy into thermal energy. From the UV-Vis spectra for microwave irradiated $1 \mathrm{mM} \mathrm{AgNO}_{3}$ solution in the presence of culture supernatants of Bacillus subtilis (Figure 3) it can be observed that the silver surface plasmon band occurs at around $410 \mathrm{~nm}$ and steadily increase in intensity as a function of time of reaction. It can be seen that surface plasmon absorption band of $\mathrm{Ag}$ particles was observed just after 5 cycles of irradiation and increased with further irradiation. The absorbance observed at $12^{\text {th }}$ cycle (180 s of irradiation) was roughly similar to that obtained by the nonmicrowave reaction incubated for $72 \mathrm{~h}$ at $40^{\circ} \mathrm{C}$. The surface plasmon band in the silver nanoparticles solution remains close to $410 \mathrm{~nm}$ throughout the reaction period, suggesting 
that the particles are dispersed in the aqueous solution with no evidence for aggregation. Not only the heating is faster through microwave radiation, but also the temperature distribution of the solution is more uniform. As such, this has led to the fast reaction rate and narrow size distribution of the Ag nanoparticles. This rapid microwave heating also provides uniform nucleation and growth conditions, leading to homogeneous nanomaterials with smaller sizes. Power dissipation is fairly uniform throughout with "deep" inside-out heating of the polar solvents, which leads to a better crystallinity.

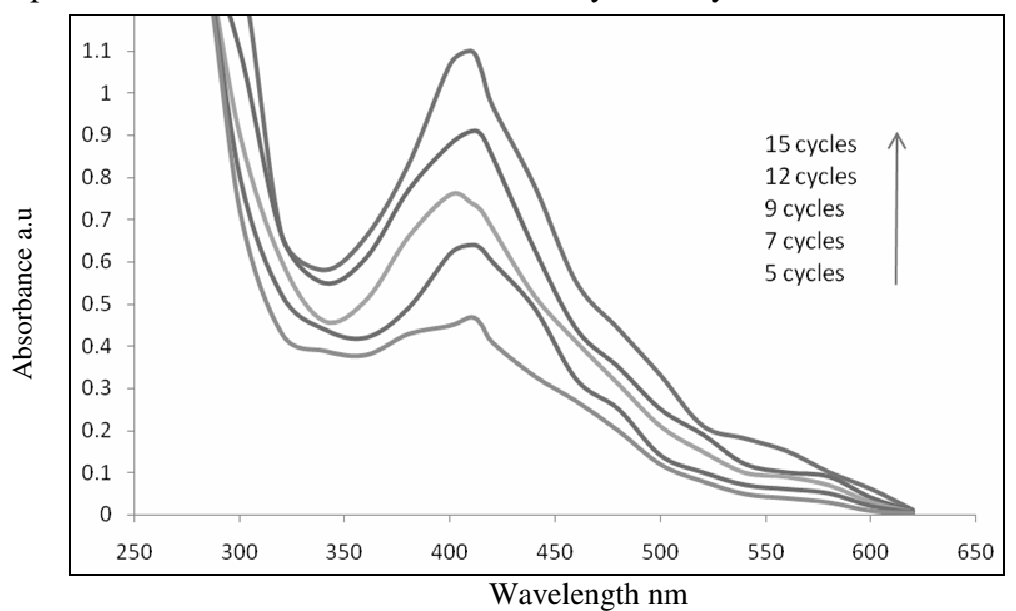

Figure 3. UV-Vis spectra recorded as a function of time of reaction of $1 \mathrm{mM}$ aqueous solution of $\mathrm{AgNO}_{3}$ with Bacillus subtilis culture supernatant irradiated with microwave. The irradiation period was 5 cycles up to 15 (microwave exposure was $15 \mathrm{~s}$ per cycle).

It can be seen from Figure 3 that the bands are sharper and more symmetrical, which reflect more uniform size distribution. The major advantage noticed is the time of reaction; the reaction proceeds at a much faster rate when irradiated with microwave.

A representative TEM picture recorded from the silver nanoparticle film deposited on a carbon coated copper TEM grid is shown in Figure 4. This picture shows individual silver particles as well as a number of aggregates. The morphology of the nanoparticles is variable, with majority of them spherical. By gentle heating, using microwave irradiation of an aqueous silver nitrate solution containing Bacillus subtilis culture supernatant, we had obtained relatively mono-disperse silver nanoparticles. Under observation of such images in an optical microscope, these assemblies were found to be aggregates of silver nanoparticles in the size range $5-50 \mathrm{~nm}$. The nanoparticles were not in direct contact even within the aggregates, indicating stabilization of the nanoparticles by a capping agent. This corroborates with the previous observation by Ahmad et $a l^{14}$ in their study on $F$. oxysporum.

As discussed earlier, the silver nanoparticle solution, synthesized by the reaction of $\mathrm{Ag}^{+}$ ions with Bacillus subtilis culture supernatant, is exceptionally stable and the stability is likely to be due to capping with proteins secreted by the bacteria. The separation between the silver nanoparticles seen in the TEM image could be due to capping by proteins and would explain the UV-Vis spectroscopy measurements, which is characteristic of welldispersed silver nanoparticles. The nanoparticles present in the aqueous medium were quite stable, even up to 5 months of incubation at $25^{\circ} \mathrm{C}$ with no signs of aggregation even at the end of this period. 


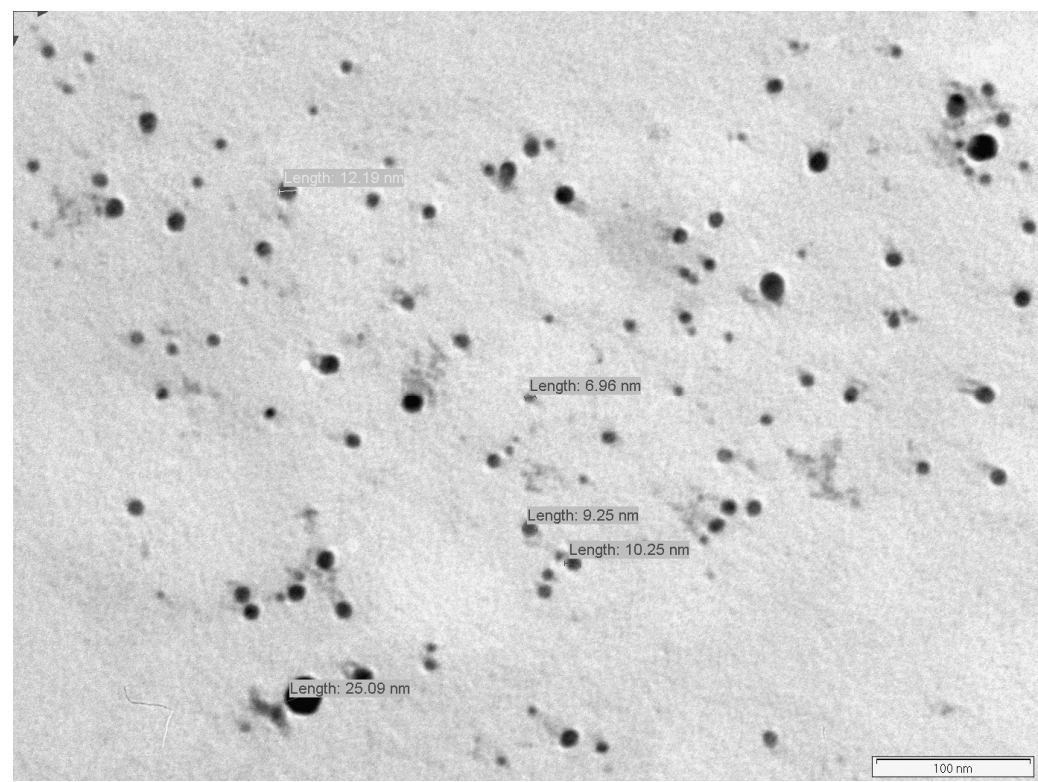

Figure 4. TEM micrograph at 100,000 times magnification recorded from a drop-coated film of $1 \mathrm{mM}$ aqueous solution of $\mathrm{AgNO}_{3}$ with Bacillus subtilis culture supernatant irradiated with microwave. The scale bar corresponds to $100 \mathrm{~nm}$.

The particles are thus stabilized in solution by the capping agent that is likely to be proteins secreted by the biomass. This is an important aspect of synthesis of nanoparticles, since the lack of sufficient stability of many nanoparticles preparation has to some extent impeded the development of the real world applications of nanomaterials.

Application of the biological systems for synthesis of silver nanoparticles has already been reported earlier ${ }^{16,}$ However, the exact reaction mechanism leading to the formation of silver nanoparticles by all these organisms is yet to be elucidated. Ahmad et $a l^{14}$ have reported that certain NADH dependent reductase was involved in reduction of silver ions in case of $F$. oxysporum. While the above experiments clearly establish that the reduction of the $\mathrm{Ag}^{+}$ions occurs extracellularly, it would be important to identify the reducing agents responsible for this. The average protein contains $3.5 \%$ phenylalanine, $3.5 \%$ tyrosine and $1.1 \%$ tryptophan: these three amino acids contribute the majority of the proteins absorbance and fluorescence properties in the $250-300 \mathrm{~nm}$ wavelength range, although if the protein contains significant numbers of disulphide bonds these too will contribute to the absorption properties in the wavelength range $250-280 \mathrm{~nm}$. On the other hand the peptide bond and other amino acids contribute to absorbance properties in the $210-220 \mathrm{~nm}$ regions. The Figure 5 shows the UV-Vis spectrum in low wavelength region recorded from the reaction of $10^{-3} \mathrm{M} \mathrm{AgNO}_{3}$ with culture supernatant of Bacillus subtilis after $12^{\text {th }}$ cycle of microwave irradiation. The presence of the absorption band around $275 \mathrm{~nm}$ is attributed to aromatic amino acids of proteins. It is well known that the absorption band at around $275 \mathrm{~nm}$ arises due to electronic excitations in tryptophan and tyrosine residues in the proteins. This observation indicates the release of proteins into solution by $B$. subtilis and suggests a possible mechanism for the reduction of the metal ions present in the solution. In this study, the UV-Vis spectrum study provides some clues regarding the mechanism of synthesis of 
silver nanoparticles. There were two absorbance peaks found in the UV range corresponding to 220 and $275 \mathrm{~nm}$. While the peak at $220 \mathrm{~nm}$ may be due to absorption by amide bond, the other peak at $275 \mathrm{~nm}$ may be attributed to the tryptophan and tyrosine residues present in the protein. This indicates secretion of some proteinic components into the medium by the bacterial biomass which might play important role in the reduction of the metal ions in the form of nanoparticles. Consequently the proteins also may bind to the nanoparticles and enhance the stability.

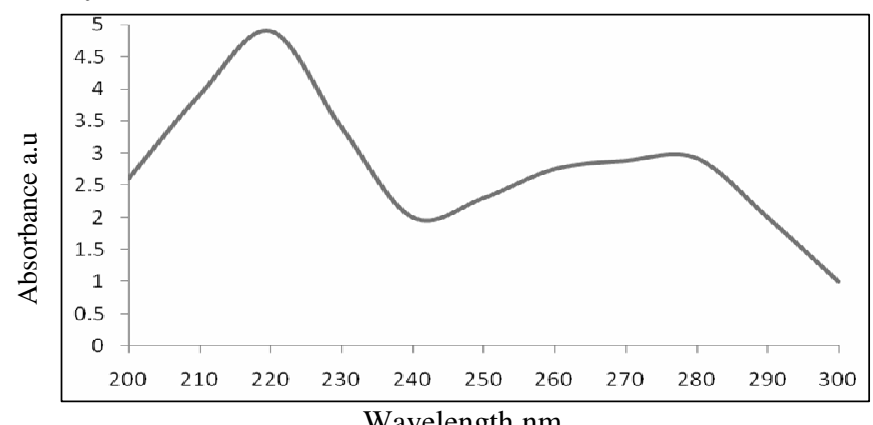

Figure 5. UV-Vis spectra in the low wavelength region recorded from the reaction of $10^{-3}$ $\mathrm{M} \mathrm{AgNO}$ with culture supernatant of Bacillus subtilis after $12^{\text {th }}$ cycle of microwave irradiation.

Previous studies ${ }^{14-16,19}$ have indicated that NADH- and NADH-dependent enzymes are important factors in the biosynthesis of metal nanoparticles. The reduction seems to be initiated by electron transfer from the NADH by NADH-dependent reductase as electron carrier. Many fungi that exhibit these characteristic properties, in general, are capable of reducing $\mathrm{Au}(\mathrm{III})$ or $\mathrm{Ag}(\mathrm{I})^{29}$. Besides these extracellular enzymes, several naphthoquinones ${ }^{31}$ and anthraquinones ${ }^{23}$ with excellent redox properties, were reported in $F$. oxysporum that could be act as electron shuttle in metal reductions ${ }^{22}$. The exact mechanism of the reduction of metal ions is yet to be elucidated for bacteria. In this study nitrate reductase activity was detected in the culture supernatant of Bacillus subtilis and was about $152 \mathrm{nmol} / \mathrm{h} / \mathrm{mL}$ culture supernatant of Bacillus subtilis. It appears that the reductase together with electron shuttling compounds and other peptides/proteins may be responsible for the reduction of $\mathrm{Ag}^{+}$ions and the subsequent formation of silver nanoparticles.

In conclusion, the Bacillus subtilis has shown potential for extracellular synthesis of fairly monodispersed, silver nanoparticles in the range of 5-50 nm. The kinetics of silver nanoparticles synthesis using the cell filtrates in combination with microwave irradiation indicates that the rapid synthesis of nanoparticles would be suitable for developing a "green nanotechnology" biosynthesis process for mass scale production. The extracellular synthesis offers a great advantage over an intracellular process of synthesis from the application point of view. Since the nanoparticles formed inside the biomass would have required additional step of processing for release of the nanoparticles from the biomass by ultrasound treatment or by reaction with suitable detergents. The extracellular synthesis of nanoparticle makes it possible to harness and immobilize/deposit onto desired solid support for the use of different practical purposes. In future, it would be important to understand the biochemical and molecular mechanism of the synthesis of the nanoparticles by the cell filtrate in order to achieve better control over size and polydispersity of the nanoparticles 


\section{References}

1. Petit C, Lixon P, and Pileni M. P, J Phys Chem., 1993, 97, 12974-12983.

2. Heath J R, Knobler C M and Leff D V, J Phys Chem., B, 1997, 101, 189-197.

3. Liz-Marzán L M and Lado-Touriňo I, Langmuir, 1996, 12, 3585-1589.

4. Komarneni S, Newalkar D Li, B, Katsuki H and Bhalla A S, Langmuir, 2002, 18, 5959-5962.

5. Santos I P and Liz-Marzán L M, Langmuir, 2002, 18, 2888-2894.

6. Harfenist S A, Wang Z L, Alvarez M M, Vezmar I and Whetten R L, J Phys Chem., 1996, 100, 13904-13910.

7. Stiger R M, Gorer S, Craft B and Penner P M, Langmuir, 1999, 15, 790-798.

8. Pol V G, Srivastava D N, Palchik O, Palchik V, Slifkin M A, Weiss A. M and Gedanken A, Langmuir, 2002, 18, 3352-3357.

9. Itakura T, Torigoe K and Esumi K, Langmuir, 1995, 11, 4129-4134.

10. Abid J P, Wark A W, Brevet P F and Girault H H, Chem. Commun., 2002, 792-793.

11. Huang H and Yang X, Carbohydr. Res., 2004, 339, 2627-2631.

12. Klaus-Joerger T, Joerger R, Olsson E and Granqvist C, Trends Biotechnol., 2001, 19(1), 15-20.

13. Mukherjee P, Ahmad A, Manda I D, Senapati S, Sainkar S R, Khan M I, Parishcha R, Ajaykumar P V, Alam M, Kumar R and Sastry M, Nano Lett., 2001, 1(10), 515-519.

14. Ahmad A, Mukherjee P, Senapati S, Mandal D, Khan M I, Kumar R and Sastry M, Coll. Surf., B, 2003, 28(4), 313-318.

15. Mukherjee P, Senapati S, Mandal D, Ahmad A, Islam Khan M, Kumar R and Sastry M, Chem Bio Chem., 2002, 3, 461-463.

16. Ahmad, A, Senapati S, Islam Khan M, Kumar R and Sastry M, Langmuir, 2003, 19, 3550-3553.

17. Gardea-Torresdey J L, Gomez E, Peralta-Videa J R, Parsons J G, Troiani H and Yacaman M J, Langmuir, 2003, 19, 1357-1361.

18. Shankar S S, Ahmad A and Sastry M, Biotechnol Prog., 2003, 19, 1627-1631.

19. Durán N, Marcato P D, Alves O L, Gabriel I H, Souza D E and Esposito E, $J$ Nanobiotechnology, 2005, 3, 1-7.

20. Mukherjee P, Ahmad A, Mandal D, Senapati S, Sainkar S R and Khan M I, Nano Lett., 2001, 1, 515-9.

21. Joerger R, Klaus T and Granqvist C G, Adv Mater, 2000, 12, 407-409.

22. Newman D K and Kolter R, Nature, 2000, 405, 94-97.

23. Baker R A and Tatum J H, J Ferment Bioeng, 1998, 85, 359-361.

24. Klaus T, Joerger R, Olsson E and Granqvist C G, Proc Natl Acad Sci USA 1999; 96, 13611-4.

25. Nair B and Pradeep T, Cryst Growth Des, 2002, 2, 293-8.

26. Zhou Y, Itoh H, Uemura T, Naka K and Chujo Y, Langmuir, 2002, 18, 277.

27. Harley S M, The American Biology Teacher 1993, 55(3), 161-164.

28. Baker R A and Tatum J H, J Ferment Bioeng, 1998, 85, 359-61.

29. Lloyd J R, FEMS Microbiol Rev., 2003, 27, 411-425.

30. Medentsev A G and Alimenko V K, Phytochemistry, 1998, 47, 935-959.

31. Harley S, The American Biology Teacher, 1993, 55, 161-164. 


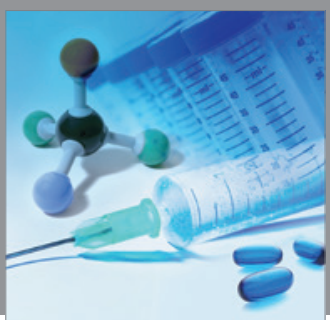

International Journal of

Medicinal Chemistry

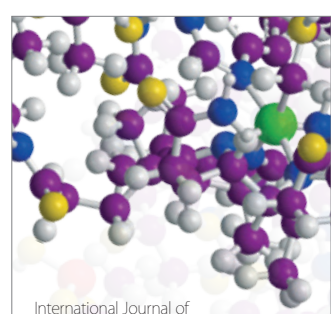

Carbohydrate Chemistry

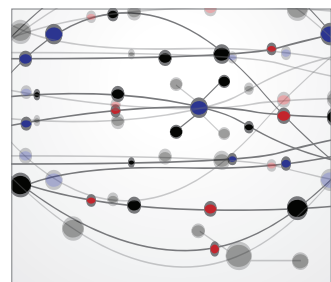

The Scientific World Journal
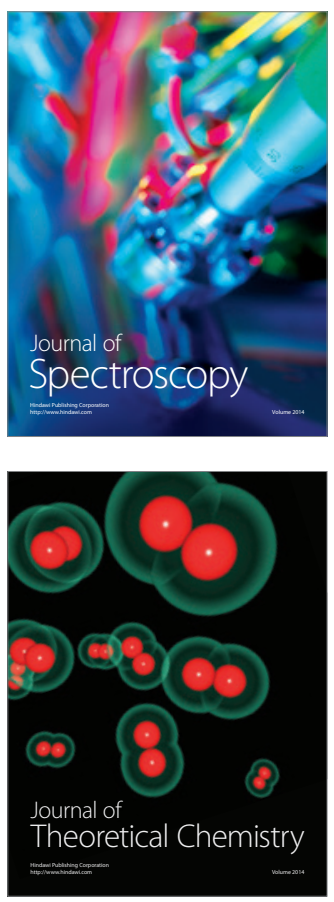
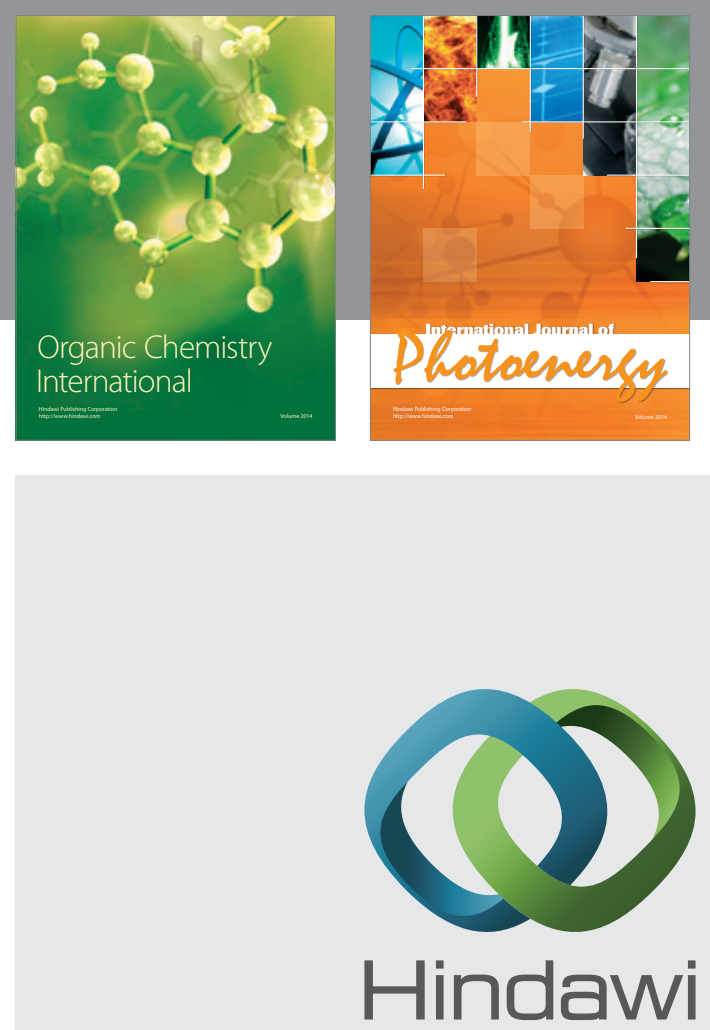

Submit your manuscripts at

http://www.hindawi.com
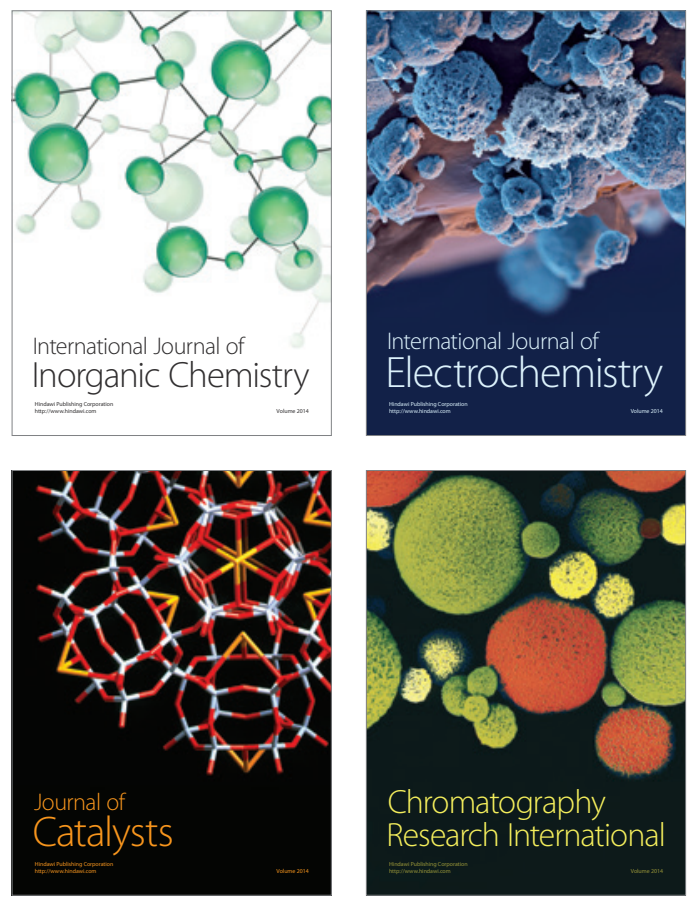
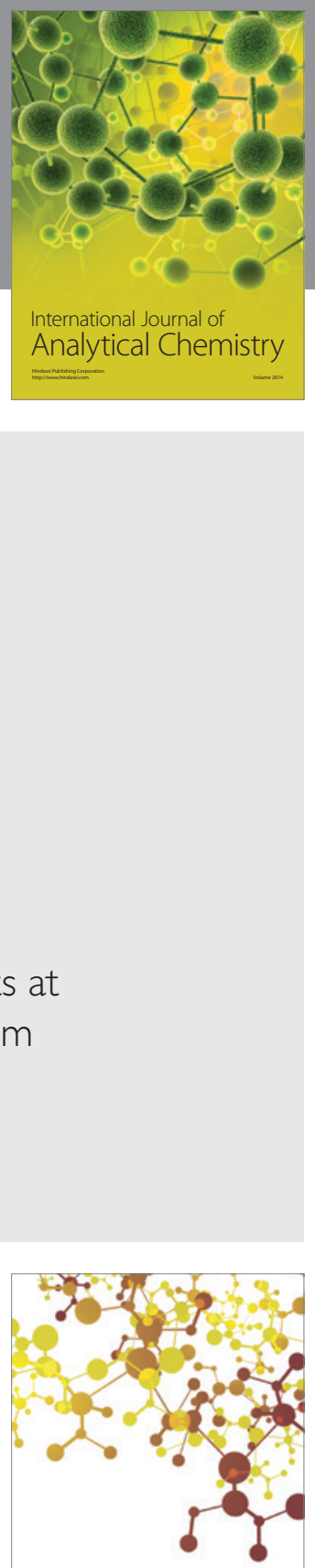

Journal of

Applied Chemistry
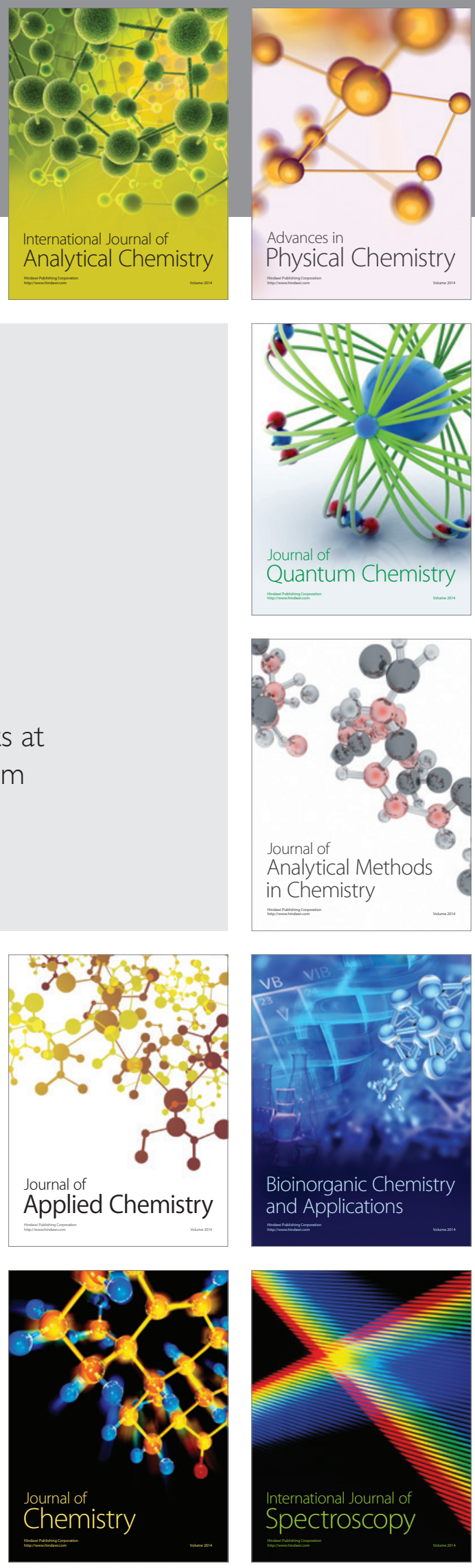